\title{
Recurrent Cystic Hygroma with Normal Karyotype in Two Consecutive Pregnancies
}

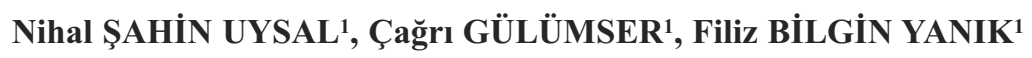 \\ Ankara, Turkey
}

\begin{abstract}
We herein describe a woman with two consecutive pregnancies affected by fetal nuchal cystic hygroma $(\mathrm{CH})$ with a normal karyotype.
\end{abstract}

A 33-year-old woman (gravidity 2, parity 1) was referred to us because of fetal hydrops. No consanguinity or Rh isoimmunization was involved in her current or previous pregnancy. First-trimester ultrasonography revealed nuchal $\mathrm{CH}$, and chorion villus sampling was performed to exclude aneuploidy.

In the first pregnancy, the $\mathrm{CH}$ had regressed and aortic coarctation was detected by second-trimester fetal echocardiography. In the current pregnancy, the $\mathrm{CH}$ had progressed and was complicated by the development of nonimmune hydrops. Termination of the pregnancy was performed at 21 weeks' gestation.

Recurrence of fetal $\mathrm{CH}$ in subsequent pregnancies is extremely rare. $\mathrm{CH}$ with a normal karyotype can be inherited as an autosomal recessive trait. This report describes a woman with recurrent $\mathrm{CH}$ with normal karyotypes and different prognoses.

Keywords: Hygroma, Cystic, Nonimmune hydrops

Gynecol Obstet Reprod Med 2016;22(3):165-167 DOI: 10.21613/GORM.2016.486

\section{Introduction}

Cystic hygroma $(\mathrm{CH})$ is an anomaly of the lymphatic system characterized by single or multiple cysts within the soft tissues, usually involving the neck. CHs are very frequently associated with chromosomal aberrations and a wide variety of anatomic defects. The incidence of nuchal $\mathrm{CH}$ is $1 / 6000$ at birth and 1/750 among spontaneous abortions (1). Recurrence of fetal $\mathrm{CH}$ in subsequent pregnancies is extremely rare.

CHs are sometimes associated with hydrops fetalis. Hydrops fetalis or fetal hydrops is a serious fetal condition defined as abnormal accumulation of fluid in two or more fetal compartments. This fluid accumulation may involve ascites, pleural effusion, pericardial effusion, and skin edema. Hydrops fetalis is mainly classified as immune or nonimmune. In recent years, nonimmune hydrops has been more frequently observed than immune hydrops; the reported prevalence in published series ranges from $1 / 1700$ to $1 / 3000$ pregnancies (2-

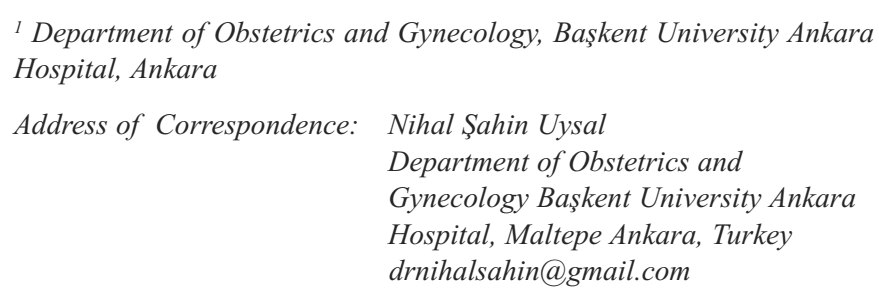

Submitted for Publication:

06. 07.2015

Accepted for Publication:
4) The pathophysiologic mechanism of hydrops fetalis is fluid extravasation and accumulation in the interstitial compartments with various causes. Nonimmune hydrops may be idiopathic in up to $25 \%$ of cases.

In this report, we describe a woman with recurrent $\mathrm{CH}$ in two fetuses with normal karyotypes and different prognoses. $\mathrm{CH}$ was complicated with hydrops fetalis in her second pregnancy.

\section{Case Report}

A 33-year-old woman (gravidity 2, parity 1) was admitted to our center at the 18 weeks 2 days of gestation because of fetal hydrops. Her first pregnancy was complicated by a fetal $\mathrm{CH}$ at 12 weeks of gestation. Chorion villus sampling revealed a 46, XY fetal karyotype. Subsequent ultrasonographic examinations during the second trimester showed that the $\mathrm{CH}$ was decreasing in size. Aortic coarctation was detected by fetal echocardiography, and the infant underwent surgical repair after birth.

The patient had no history of chronic medical disorders or teratogen exposure during her current pregnancy. There was no consanguinity or $\mathrm{Rh}$ isoimmunization. During the first trimester, chorion villus sampling was performed because of increased nuchal translucency and revealed a normal karyotype. An ultrasonographic examination at 18 weeks of gestation was consistent with hydrops fetalis based on the presence 
of a $\mathrm{CH}$ behind the neck ( $37 \mathrm{~mm}$ in thickness), ascites, pleural effusion, and edematous subcutaneous tissue. Fetal biometric measurements were consistent with the gestational age of 17 weeks (Figure 1). A maternal indirect Coombs test, and serological screening for intrauterine infections were negative. Fetal echocardiography showed normal cardiac anatomy. Ultrasonographic reevaluation was performed 1 week later; the subcutaneous edema had increased (11 $\mathrm{mm}$ in thickness) and the amniotic fluid in one vertical pocket measured $22 \mathrm{~mm}$ (Figure 2). Doppler evaluation of the ductus venosus revealed a reversed "a wave," indicating cardiac failure (Figure 3 ). The fetal biometric measurements were not consistent with the gestational age as determined by the patient's last menstrual period, but were instead consistent with a gestational age of 2 weeks earlier. The parents were instructed according to these findings. They opted for follow-up of the pregnancy. At the next visit (21 weeks of gestation), the ultrasonographic findings were similar and the couple opted for termination of the pregnancy, which was performed by vaginal administration of misoprostol. A male fetus weighing $910 \mathrm{~g}$ was delivered.

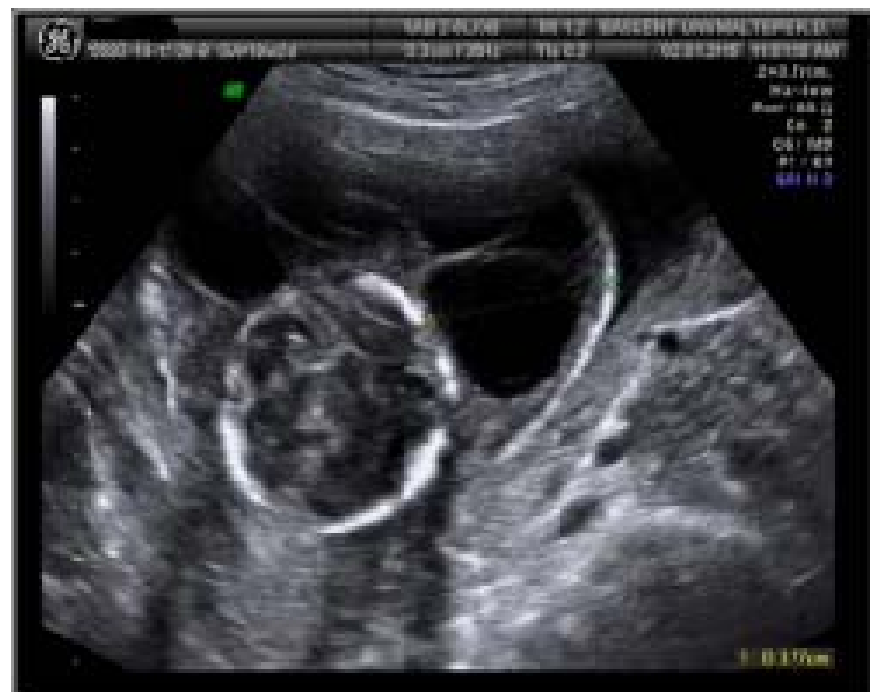

Figure 1: Cystic hygroma behind the neck, $37 \mathrm{~mm}$ thick

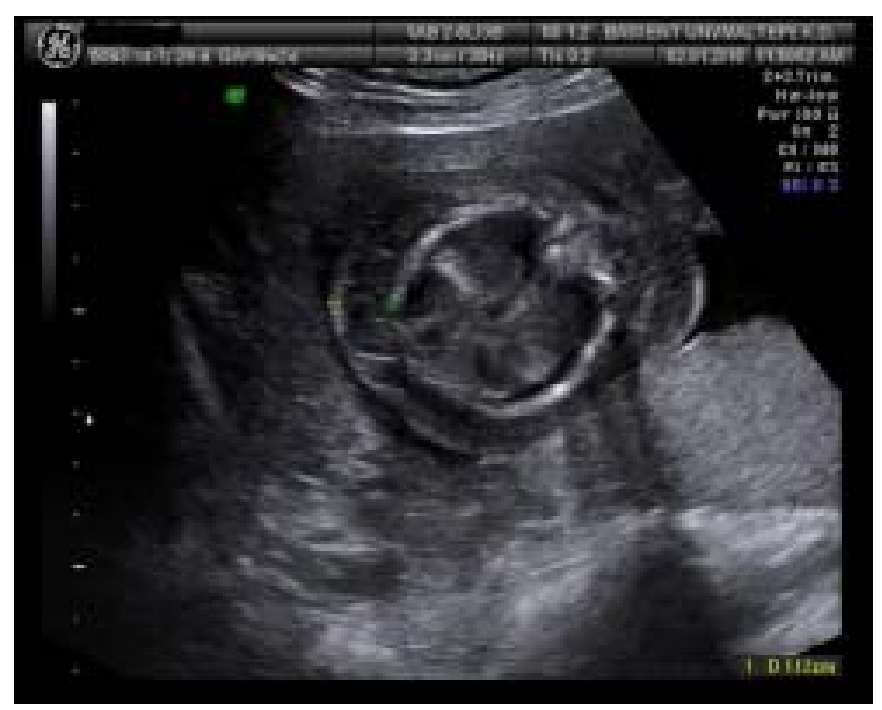

Figure 2: Subcutaneous edema, $11 \mathrm{~mm}$ thick

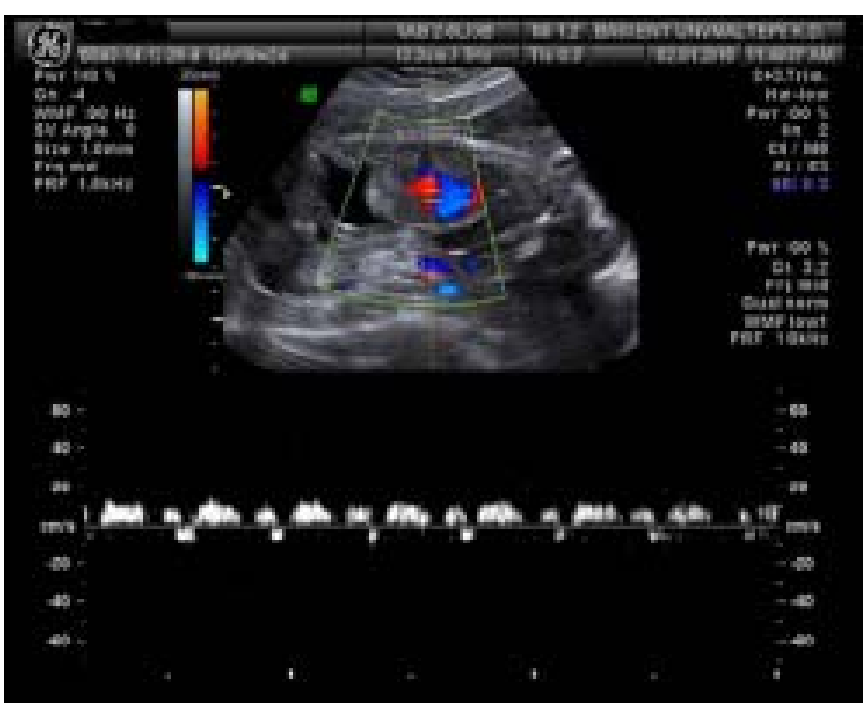

Figure 3: Doppler evaluation of the ductus venosus revealed an "a wave"

Pathological examination of the placenta revealed a large placenta for the gestational age, edematous chorionic villi, and a few focal avascular villi. Hydrops fetalis, a $\mathrm{CH}$, and fetal biometric measurements compatible with a gestational age of 17 weeks were also verified by postpartum autopsy.

\section{Discussion}

Recurrent fetal $\mathrm{CH}$ in two or more pregnancies is an extremely rare condition based on reports in the literature. If fetal $\mathrm{CH}$ is diagnosed, the initial work-up procedure should be fetal karyotyping because of the possible association with chromosomal abnormalities. $\mathrm{CH}$ associated with a normal karyotype can be inherited as an autosomal recessive trait $(5,6)$. To determine the fetal outcome in cases of $\mathrm{CH}$ with normal karyotypes, detailed sonographic examination should be performed to exclude fetal heart defects as well as skeletal, urogenital, and craniofacial anomalies because these might cause severe morbidity (7). Even if the fetal karyotype and detailed ultrasonographic evaluation appear to be normal in some cases of $\mathrm{CH}$, chromosomal microarray analysis is a promising method for future evaluation.

$\mathrm{CH}$ can progress and become complicated with nonimmune hydrops, as in the present case. Nonimmune hydrops fetalis may result from fetal intrauterine infections, hematological problems, thoracic problems, lymphatic dysplasia, and genetic syndromes. Placental pathologies can also be associated with hydrops. Other rare causes of hydrops fetalis are fetal tumors, metabolic and neurological diseases, gastrointestinal anomalies, and mitochondrial mutations (8).

The work-up for hydrops fetalis should include a maternal medical and obstetric history, detailed sonographic examination, Doppler studies, fetal echocardiography, indirect Coombs test, fetal karyotyping, maternal serological screening, genetic counseling with or without genetic microarray, 
and amniotic polymerase chain reaction (PCR) studies to identify infection markers. X-ray or magnetic resonance imaging of the fetus, placental pathology and autopsy can give information about the etiology and may be helpful in subsequent pregnancies (8). The etiology cannot be detected in $15 \%$ to $25 \%$ of cases of nonimmune hydrops fetalis.

The occurrence of $\mathrm{CH}$ in two consecutive pregnancies in the present case is interesting. In the first pregnancy, the $\mathrm{CH}$ had regressed; there was no hydrops, but coarctation of the aorta was present. In the second pregnancy, the $\mathrm{CH}$ had progressed and was associated with hydrops. Other etiological factors were excluded; thus, the only problem leading to hydrops fetalis appeared to be the $\mathrm{CH}$ itself. $\mathrm{CH}$ is associated with abnormal lymphatic drainage in that more fluid accumulates in the extravascular space than is resorbed into the intravascular space. $\mathrm{CH}$ may be a part of generalized lymphatic dysplasia, or it may cause cardiac dysfunction due to decreased preload, leading to an end result of nonimmune hydrops fetalis (9). When there is no fetal structural anomaly, genetic syndrome, or abnormal karyotype, the $\mathrm{CH}$ is not complicated by hydrops and generally regresses with a better prognosis (10).

\section{References}

1. Chen CP, Liu FF, Jan SW, Lee CC, Town DD, Lan CC. Cytogenetic evaluation of cystic hygroma associated with hydrops fetalis, oligohydramnios or intrauterine fetal death: the roles of amniocentesis, postmortem chorionic villus sampling and cystic hygroma paracentesis. Acta Obstet Gynecol Scand 1996 May;75(5):454-8.
2. Heinonen S, Ryynänen M, Kirkinen P. Etiology and outcome of second trimester non-immunologic fetal hydrops. Acta Obstet Gynecol Scand 2000;79(1):15-8.

3. Hutchison AA, Drew JH, Yu VY, Williams ML, Fortune DW, Beischer NA. Nonimmunologic hydrops fetalis: a review of 61 cases. Obstet Gynecol 1982;59(3):347-52.

4. Machin GA. Hydrops revisited: literature review of 1.414 cases published in the 1980s. Am J Med Genet 1989;34 (3):366-90.

5. Baxi L, Brown S, Desai K, Thaker H. Recurrent cystic hygroma with hydrops. Fetal Diagn Ther 2009;25(1):127-9.

6. Teague KE, Eggleston MK, Muffley PE, Gherman RB. Recurrent fetal cystic hygroma with normal chromosomes: case report and review of the literature. J Matern Fetal Med 2000 Nov-Dec;9(6):366-9.

7. Tanriverdi HA, Ertan AK, Hendrik HJ, Remberger K, Schmidt W. Outcome of cystic hygroma in fetuses with normal karyotypes depends on associated findings. Eur J Obstet Gynecol Reprod Biol 2005;118(1):40-6.

8. Desilets V, Audibert F. Investigation and management of non-immune fetal hydrops. J Obstet Gynaecol Can 2013;35(10):923-38.

9. Apkon M. Pathophysiology of hydrops fetalis. Semin Perinatol 1995;19(6):437-46.

10. Beke A, Joó JG, Csaba A, Lázár L, Bán Z, Papp C, et al. Incidence of chromosomal abnormalities in the presence of fetal subcutaneous oedema, such as nuchal oedema, cystic hygroma and non-immune hydrops. Fetal Diagn Ther 2009;25(1):83-92. 